5. O'Malley KJ, Pound CR, Walsh PC, Epstein JI, Partin AW: Influence of biopsy perinerual invasion on long-term biochemical disease-free survival after radical prostatectomy. Urology. 2002; 59: 85-90.

Dr. Athanase Billis

Full-Professor of Pathology

State University of Campinas, Unicamp

Campinas, São Paulo, Brazil

E-mail: athanase@fcm.unicamp.br

doi: $10.1590 / S 1677-553820100001000024$

\title{
Transurethral resection specimens of the bladder (TURB): Outcome of invasive urothelial cancer involving muscle bundles indeterminate between muscularis mucosae and muscularis propria
}

H Miyamoto, JI Epstein

The Johns Hopkins Hospital, Baltimore, United States

Mod Pathol 2010;in press [Abstract from the USCAP meeting, 2010]

Background: It may be difficult to diagnose muscularis propria on TURB as thin muscle fibers on TURB may represent either muscularis propria destroyed or splayed by urothelial carcinoma or muscularis mucosae, which may be hyperplastic.

Design: 95 invasive bladder cancers seen at our instituion (1986-2008) with follow-up (mean 25.4 months) where the initial TUR pathologic stage was ambiguous (T1 vs. T2) were analyzed (73 men; 22 women; mean age 69.4 years).

Results: Subsequent restaging TURB or definitive therapeutic procedures performed $\leq 3$ months after the original TURB done in 58 cases revealed $22(37.9 \%)$ patients with non-muscle invasive disease and $32(55.2 \%)$ patients with $\geq$ pT2 disease. Staging in 4 cases remained ambiguous. 37 cases eventually developed $\geq$ pT2 disease in 2/22 (9.1\%) cases with non-muscle invasive disease on initial restaging TURB, 2/4 (50.0\%) of cases with uncertain stage disease, and 14/37 (37.8\%) cases with no restaging TURB. Patients with a final stage of non-muscle invasive disease had a lower risk of progression (T4 or metastatic disease) vs. those with a final stage of $\geq \mathrm{pT} 2(\mathrm{p}=0.003)$, uncertain stage $(\mathrm{p}=0.012)$, or no stage confirmation $(\mathrm{p}=0.043)$.

Conclusions: This is the first study to evaluate follow-up when initial TURB is equivocal for muscularis propria invasion. Similar to an atypical prostate needle biopsy, urologists should be encouraged to perform restaging TURBs in cases of equivocal muscularis propria invasion. Although this may seem intuitive, 37/95 cases did not have repeat staging/therapeutic procedures done within 3 months of initial TURB; $37.8 \%$ of these patients eventually developed $\geq \mathrm{T} 2$ disease.

\section{Editorial Comment}

It is of utmost importance the staging of urothelial carcinomas of the urinary bladder. In stage pT2 (invasion of the muscularis propria) is indicated radical cystectomy. Sometimes the distinction between muscularis mucosae and muscularis propria is a dilemma for the pathologist. Invasion of the muscularis mucosa is stage pT1.

Morphologically these two muscular layers are distinct. In muscularis mucosa, the fibers are thin and spaced; in muscularis propria, the fibers form compact aggregates. It is interesting to note that description of the muscularis mucosae will not be found in Histology texts. The existence and morphology of this layer was 
described in 1983 by Dixon and Gosling (1) and the importance for staging and treatment of bladder urothelial carcinoma by Ro JY et al (2) from the MD Anderson Hospital in Houston.

In some cases it is difficult if not impossible for the pathologist to recognize that the invaded muscular layer is the muscularis mucosae. This happens because the fibers of this layer may be thick due to hypertrophy. In doubt, the pathologist should always ask for a restaging TUR of the bladder. Another much commoner condition for asking a restaging TUR is whenever the specimen does not contain muscularis propria.

\section{References}

1. Dixon JS, Gosling JA: Histology and fine structure of the muscularis mucosa of the human urinary bladder. J Anat. 1983; 136: 265-71.

2. Ro JY, Ayala AG, El-Naggar A: Muscularis mucosa of urinary bladder: importance for staging and treatment. Am J Surg Pathol. 1987; 11: 668-73.

Dr. Athanase Billis

Full-Professor of Pathology State University of Campinas, Unicamp

Campinas, São Paulo, Brazil

E-mail: athanase@fcm.unicamp.br 